\title{
Os serviços abertos de Saúde Mental no Brasil: o cuidado em liberdade na perspectiva dos higienistas
}

\author{
Carina Furlaneto Frazatto*1 \\ Marina Maria Beltrame*2
}

O objetivo deste estudo é apresentar considerações de alguns médicos higienistas sobre os serviços abertos de saúde mental desenvolvidos no Brasil no início do século XX. Analisamos publicações dos Archivos Brasileiros de Hygiene Mental e outros materiais (1925-1955) e verificamos que a possibilidade de cuidar em liberdade (defendida pela atual Reforma Psiquiátrica) já era vislumbrada pelos higienistas, em um período no qual a assistência era, hegemonicamente, pautada no isolamento em instituições psiquiátricas.

Palavras-chaves: Higiene Mental, História da Psiquiatria, Reforma

Psiquiátrica, Serviços de Saúde Mental

*1 Pontifícia Universidade Católica de São Paulo - PUC-SP (São Paulo, SP, Br).

*2 Universidade Estadual de Maringá - UEM (Maringá, PR, Br). 
Este trabalho enfoca os serviços abertos de saúde mental no Brasil, a partir do resgate das considerações debatidas pelos higienistas no início do século XX. De forma sucinta, podemos pontuar que os higienistas ${ }^{1}$ eram médicos e intelectuais em geral, integrantes da Liga Brasileira de Higiene Mental (LBHM), entidade civil fundada em 1923, que tinha entre as suas finalidades a prevenção das enfermidades mentais, o amparo aos egressos dos manicômios e a "melhoria progressiva nos meios de assistir e tratar os doentes nervosos e mentais em asilos públicos, particulares ou fora deles" (Liga Brasileira de Hygiene Mental, 1925, p. 223). Pautados nos conceitos das ciências naturais, os higienistas buscaram solucionar os problemas sociais da época — desde doenças físicas até os costumes e comportamentos considerados desviantes pela sociedade burguesa - por meio da higienização física e mental da população, propondo intervenções em diversos âmbitos, tais como na educação e na saúde. A base psiquiátrica desses profissionais continha uma tendência a associar certos problemas psiquiátricos a questões culturais mais gerais relativas ao povo brasileiro, atribuindo-os, por exemplo, à situação social dos imigrantes ou à constituição étnica dos brasileiros (Costa, 2007). Ao transporem de forma direta os preceitos das ciências naturais às ciências sociais, os higienistas concebiam, muitas vezes, as diferenças sociais como resultantes da natureza humana e não do modo como os homens constroem as relações entre eles. Entretanto, tal concepção não deve ofuscar as contribuições desses intelectuais que se dedicaram à saúde mental e consideraram, dentro daquele contexto, propostas que poderiam melhorar a assistência nesta área.

\footnotetext{
${ }^{1}$ Para mais informações sobre os higienistas, sugerimos os textos de Costa (2007) e Boarini (2003).
} 
Para discutirmos essa temática, utilizamos como fonte de dados o acervo bibliográfico do Grupo de Estudos e Pesquisas sobre o Higienismo e o Eugenismo (GEPHE), as publicações Archivos Brasileiros de Hygiene Mental, Archivos Paulistas de Hygiene Mental e Boletim de Hygiene Mental. A nosso ver, a recuperação das intervenções propostas pelos higienistas permite delinear um panorama de como se configurou os primórdios da assistência em meio aberto no Brasil, além de levantar elementos para uma reflexão sobre as práticas vigentes no âmbito da saúde mental.

A expressão "serviços abertos", utilizada pelos higienistas nas fontes pesquisadas, refere-se aos serviços de assistência em saúde mental que não se baseavam no isolamento como prática terapêutica. Mas em que consistiam essas propostas há quase um século? Mantinham alguma semelhança com os serviços atuais? Podem ser consideradas o embrião da atual Reforma Psiquiátrica brasileira?² Ao voltarmos na história, observamos que muitas décadas antes do início da Reforma Psiquiátrica, em um contexto no qual o modelo asilar era hegemônico, surgiam as primeiras considerações sobre a possibilidade de atendimento em meio aberto. Ainda que não fosse unanimidade entre os higienistas da época, a superlotação dos manicômios brasileiros, os gastos com a manutenção das pessoas internadas nos hospitais, assim como a cronificação que o regime fechado provocava, fomentaram a defesa pela criação de outras formas de atendimento às pessoas consideradas doentes mentais (Santos, 1931). São as considerações dos higienistas sobre tais formas de atendimento que descreveremos neste texto.

\section{Os serviços abertos em saúde mental sob a ótica higienista: as modalidades de atendimento e suas características}

Uma das modalidades de atendimento alternativa ao asilamento, proposta no início do século XX, refere-se à assistência familiar, forma de

\footnotetext{
${ }^{2}$ Fruto de diversos movimentos sociais brasileiros, intensificados a partir da década de 1980 , a reforma psiquiátrica brasileira visa, em linhas gerais, assegurar os direitos legais às pessoas com sofrimento psíquico e a substituição progressiva do hospital psiquiátrico por um modelo de atenção formado por uma rede integrada de serviços de base comunitária. Para se aprofundar neste assunto, sugerimos a leitura de Amarante (1995; 1996).
} 
tratamento apregoada já em 1903, conforme consta no Art. $3^{\circ}$ do Decreto n. 1.132 de 22 de dezembro: "O enfermo de alienação mental poderá ser tratado em domicílio, sempre que lhe forem subministrados os cuidados necessários" (Brasil, 1903). ${ }^{3}$ Gustavo de Rezende ${ }^{4}$ (1932) explica que essa forma de tratamento poderia ser ofertada tanto pela própria família do enfermo quanto por famílias estranhas. No primeiro caso, eram denominadas de assistência homofamiliar e, no segundo, de assistência heterofamiliar. Devido à necessidade de conhecimentos específicos, a preferência era que essa assistência fosse prestada por famílias de funcionários dos estabelecimentos onde os pacientes estivessem internados. Além disso, as famílias deveriam residir nas proximidades do manicômio, sobretudo por duas razões: a possibilidade de recaídas e pela "facilidade de vigilância, médica e administrativa" (Rezende, 1932, p. 134).

Rezende (1932) menciona que, no Brasil, a primeira assistência familiar foi inaugurada por Francisco Franco da Rocha em 14 de abril de 1908, na cidade de São Paulo, mas é a experiência do Rio de Janeiro que o autor aborda de forma mais detalhada. Nessa cidade, o serviço de assistência heterofamiliar, cujas instalações eram anexas à Colônia de Alienadas no Engenho de Dentro, ${ }^{5}$ teve início em 1920 por iniciativa de Gustavo Riedel ${ }^{6}$ (1884-1934), psiquiatra fundador e primeiro presidente da Liga Brasileira de Higiene Mental, que dedicou sua carreira a reformar a assistência psiquiátrica. Tal serviço era constituído de 11 pavilhões, cada um deles contendo um quarto com dois leitos reservados às pacientes que ali iriam residir. De modo geral, o funcionamento desse serviço tinha os seguintes moldes:

${ }^{3}$ Esse decreto representa o primeiro registro legislativo federal direcionado aos "alienados" e tinha como objetivo reorganizar a assistência a eles dispensada (Costa, 2007).

${ }^{4}$ Gustavo de Rezende, considerado um dos pioneiros da neuropsiquiatria infantil, foi membro titular da Liga Brasileira de Higiene Mental e psiquiatra do Hospital Colônia de Psicopatas (mulheres) no Engenho de Dentro, em meados de 1934.

${ }^{5}$ Criada a partir do Decreto n. 8.834 de 11 de julho (Brasil, 1911), a Colônia objetivava melhorar a assistência aos alienados, sobretudo do sexo feminino, devido à superlotação do Hospital Nacional de Alienados no Rio de Janeiro.

${ }^{6}$ Gustavo Kohler Riedel concluiu o curso de medicina no Rio de Janeiro em 1908. Dentre as funções que exerceu durante sua vida profissional destacamos sua nomeação, em 1911, como diretor interino do Laboratório de Anatomia Patológica do Hospital Nacional de Alienados e, em 1918, sua assunção ao cargo de diretor da Colônia de Alienados do Engenho de Dentro, onde criou o Ambulatório Rivadávia Corrêa. Outras informações podem ser obtidas em: $<$ http://arch. coc.fiocruz.br/index.php/gustavo-kohler-riedel>. 


\section{HISTÓRIA DA PSIQUIATRIA}

Essas enfermeiras habitarão gratuitamente essas casas, e sem despesas com as doentes que lhe são confiadas. $\mathrm{O}$ alienista designado para dirigir a assistência heterofamiliar dá instruções aos nutrícios sobre a maneira de cuidar das doentes e exerce uma fiscalização rigorosa quanto ao cumprimento das ordens expedidas, além do exame mental e direto das pacientes que se acham na assistência, mensalmente e em qualquer ocasião que julgar oportuno. (Rezende, 1932, p. 135)

Para Ernani Lopes ${ }^{7}$ (1930), quando realizada antes da internação no manicômio, a assistência heterofamiliar traria benefícios na medida em que pouparia a pessoa do estigma da internação. Da mesma forma, Rezende (1929) destaca que o tratamento por meio da assistência familiar seria menos estigmatizante do que aquele realizado em um manicômio: "O que é ainda digno de lástima é que continua a ser julgado perigoso e incapaz de exercer qualquer função todo aquele que no seu curriculum vitae tiver um estágio num manicômio" (p. 64).

Não obstante o caráter alternativo dessa modalidade de atendimento, o objetivo de sua implementação não era, conforme pontua Jorge (1997), o questionamento do aparato manicomial como forma de cuidado, mas sim a redução de gastos e o controle da população de internos. Nas palavras do psiquiatra Juliano Moreira $^{8}$ (1906, p. 28-29, citado por Jorge, 1997): “As colônias familiares não aniquilam de nenhum modo os estabelecimentos existentes, não constituem a estada conveniente para todas as espécies de alienados, porém podem parar de modo prático, ativo e pouco dispendioso o crescimento incessante desses estabelecimentos".

Outra proposta de tratamento instalada no país nas primeiras décadas do século XX era o chamado "posto de psicopatas" (termo então usado para designar genericamente os doentes mentais). Tinha em comum com a

${ }^{7} \mathrm{O}$ material pesquisado indica que Ernani Lopes (1885-?) foi médico integrante da LBHM e seu presidente em meados de 1930, e discutiu temas como alcoolismo, eugenia, infância e higiene mental.

${ }^{8}$ De acordo com o Dicionário Histórico-Biográfico das Ciências da Saúde no Brasil (1832-1930), Juliano Moreira (1873-1933), formado na Faculdade de Medicina da Bahia, foi nomeado diretor do Hospício Nacional de Alienados em 1903, onde criou novas enfermarias, como a infantil, aboliu os coletes e camisas de força, implantou várias oficinas (artísticas, de carpintaria, de marcenaria, por exemplo), dentre outras coisas. Também propôs a substituição do hospício por diferentes formatos institucionais: hospitais urbanos para curtos períodos de internação e tratamento, hospitais-colônia, assistência heterofamiliar para casos mais longos. 
assistência familiar funcionar como um serviço aberto e a busca por superar o problema da superlotação nos manicômios. Inaugurado em 1928 no município de Porto Alegre e com suas atividades encerradas dez anos depois, sem motivo conhecido, o "posto de psicopatas" tinha como objetivo fundamental evitar que esses permanecessem nas cadeias públicas enquanto aguardavam liberação para a hospitalização no Hospício São Pedro, ${ }^{9}$ conforme análise do médico Jacintho Godoy (1955). Além de evitar a permanência dos doentes mentais nas prisões, segundo esse médico, o "posto de psicopatas" tinha outras finalidades, tais como: melhorar as instalações e a assistência aos doentes visando evitar sua internação, realizar uma triagem dos casos passíveis de cura fora do Hospício São Pedro e "promover a cura fora do Hospital São Pedro, com proveito para este Estabelecimento, que tem uma superpopulação, e para o doente que evita a tara social da internação, de grande percentagem de acidentes mentais agudos, eminentemente transitórios" (Godoy, 1955, p. 106).

É preciso lembrar que, apesar de proibida no Brasil ao menos desde 1903 (Decreto n. 1.132, 1903), a passagem de alienados pelas cadeias públicas era uma prática comum nos diversos municípios brasileiros. A título de exemplo, Godoy (1955) cita a situação da cidade de São Paulo, onde cerca de mil alienados permaneceriam "recolhidos nas cadeias do interior por falta de lugar no Hospital de Juquery" (p. 104), ${ }^{10}$ de acordo com mensagem proferida pelo Presidente do Estado de São Paulo, Antônio Dino da Costa Buengo, ao Congresso Legislativo em 1928.

Além dessa questão, o psiquiatra Antonio Carlos Pacheco e Silva ${ }^{11}$ (1928) aponta que o atendimento psiquiátrico estava condicionado a uma

${ }^{9}$ O Hospício São Pedro, identificado atualmente como Hospital Psiquiátrico São Pedro, localiza-se em Porto Alegre-RS e foi fundado em 1884. Um dos seus primeiros diretores foi o médico Jacintho Godoy (1886-1959), o qual assumiu em 1924 a direção do Manicômio Judiciário e, dois anos depois, a do Hospício São Pedro. É considerado responsável por difundir novas ideias na prática psiquiátrica, especialmente a partir de seu trabalho no citado hospital. Para mais detalhes sobre a história do Hospício São Pedro ver Wadi (2002).

${ }^{10}$ Ainda em funcionamento, mas com número reduzido de internos, o Hospital do Juquery, localizado na região metropolitana de São Paulo, teve suas atividades iniciadas em 1898, sofreu grandes ampliações até 1927, abrigando dependências femininas, masculinas, de "menores" e Manicômio Judiciário. Para um aprofundamento na história do Juquery, ver Cunha (1986).

${ }^{11}$ Antonio Carlos Pacheco e Silva (1898-1988), psiquiatra brasileiro, foi o segundo diretor do Hospital do Juquery entre os anos de 1923 e 1937 (Cunha, 1986). Editou publicações na área da psiquiatria, dentre elas o Arquivo Paulista de Higiene Mental (1928-1930). 


\section{HISTÓRIA DA PSIQUIATRIA}

guia de qualificação emitida pela polícia ou a portarias determinadas por alguma autoridade. Por essa razão, exaltou a sanção de uma lei de autoria do também médico Afrânio Peixoto, ${ }^{12}$ o Decreto n. 5.148-A de 10 de janeiro de 1927, que reorganizava a assistência a psicopatas no Distrito Federal. Esse decreto facultava a criação de serviços abertos para as pessoas denominadas naquele período como "alienados" e, a partir de então, como "psicopatas". Para Pacheco e Silva (1928), a medida poderia proporcionar os seguintes benefícios:

A lei Afrânio Peixoto veio, pois, elevar o doente da mente à categoria de "doente" sim, mas à dos "outros doentes" que entram e saem facilmente dos hospitais, sem dar contas a ninguém. Só quando alienados, isto é, perigosos a si e a outrem, serão declarados tais e submetidos ao regime jurídico, agora de exceção, até a pouco, generalizado. Além dos doentes que acorrerão a hospitais abertos, ambulatórios, dispensários, haverá visitadoras que irão aos lares descobrir os passíveis de trato especial de uma vez, é o tratamento e é a profilaxia da insanidade. É a saúde e é a justiça ao louco. (p. 2)

Para concretizar aquilo que a lei preconizava, focalizando suas reflexões sobre a assistência a alienados na cidade de São Paulo, Pacheco e Silva vislumbrava a necessidade de criação de clínicas psiquiátricas, de ambulatórios anexos aos hospitais de clínica geral e, ao lado do Hospital do Juquery, a construção de um pavilhão destinado aos doentes voluntários, "cujas perturbações não exijam a reclusão forçada nem o regime em parte fechada" (p. 5). Para o autor, tais serviços coexistiriam com os hospitais psiquiátricos. Estes últimos visariam "o tratamento das psicopatias graves, cuja internação se processe compulsoriamente", enquanto os ambulatórios serviriam "para atenderem aos psicopatas compatíveis com o meio social" (Pacheco e Silva, 1944, p. 2).

Destacamos os ambulatórios, portanto, como outra modalidade de serviço aberto. Um exemplo representativo foi o Ambulatório Rivadavia Corrêa, instalado na Colônia de Alienadas do Engenho de Dentro em 1918, por iniciativa de Gustavo Riedel. Além do acompanhamento do doente, esse serviço tinha como objetivo a prevenção dos distúrbios mentais (Jorge, 1997).

${ }^{12}$ Informações presentes no Dicionário Histórico-Biográfico das Ciências da Saúde no Brasil (1832-1930), apontam que Júlio Afrânio Peixoto (1876-1947) formou-se na Faculdade de Medicina da Bahia e integrou a LBHM. Entre os anos de 1924 e 1930, foi deputado federal e elaborou o citado Decreto n. 5.148-A de 10 de janeiro de 1927. Para maiores informações sobre o trabalho desse higienista sugerimos a leitura de Silva (2014). 
Sobre os ambulatórios, Ernani Lopes (1930) indica que, entre suas funções mais relevantes estariam as de "procurar no meio social os predispostos à neuropatia e vigiar os egressos dos Hospitais Psiquiátricos" (p. 145).

A situação dos egressos do manicômio foi outro problema que integrou o rol de preocupações da LBHM. A fim de garantir assistência a esse público, a Liga defendia oito medidas, dentre as quais destacamos:

$1^{\circ}$ Ter sempre uma relação dos parentes, amigos e conhecidos dos internados para que aqueles sejam sempre informados do estado dos pacientes e aconselhados a retirá-los do estabelecimento em caso de cura ou melhora acentuada. $2^{\circ}$ Auxiliar materialmente os egressos sem parentes ou amigos ou pessoas que por eles se interessem. $3^{\circ}$ Auxiliar materialmente os egressos cujas famílias forem necessitadas. $4^{\circ}$ Fazer o possível para arranjar colocação para os egressos válidos, de acordo com suas aptidões, em casas particulares, oficinas etc. $5^{\circ}$ Colocar os egressos incapazes de trabalhar, mas que também não podem ser conservados no serviço nem na família, em asilos, colônias familiares, ou instituições análogas. $6^{\circ}$ Estar sempre em contato com os egressos por intermédio das visitadoras sociais. $7^{\circ}$ Vulgarização dos conhecimentos de psiquiatria, de modo que o povo compreenda seu dever de auxiliar os egressos, aceitando-os na comunidade e amparando-os. $8^{\circ}$ Facilitar o tratamento, em domicílio ou em estabelecimento apropriado, dos egressos que apresentarem quaisquer manifestações que façam suspeitar a volta do estado mental patológico. (Rezende, 1929, p. 65-66)

Pelo teor dessas propostas, notamos que a atenção dedicada aos egressos esbarrava em algumas situações que iam além da patologia mental, tais como a ausência de parentes ou amigos para amparo dos mesmos, a falta de recursos materiais ou, ainda, a dificuldade da sociedade em aceitar as pessoas com transtorno mental no convívio social, o que exigia a inclusão dos diversos setores sociais na responsabilidade pelo cuidado dos egressos como o serviço das visitadoras sanitárias, as instituições com condições de ofertar trabalho, a família e a comunidade em geral.

Nessa perspectiva, encontramos no Boletim de Higiene Mental de 1949, menção à necessidade de criação de Centros de Readaptação, compostos basicamente por médicos psiquiatras e assistentes sociais psiquiátricas, cuja função seria "devolver à sociedade, no momento oportuno, o egresso do hospital psiquiátrico" (Anônimo, 1949, p. 2) $\cdot{ }^{13}$ A justificativa para a implantação desse

${ }^{13}$ Constam apenas as iniciais do autor, H. N. T., que não pudemos identificar. 


\section{HISTÓRIA DA PSIQUIATRIA}

serviço respaldava-se na observação de que o longo período de internação no hospital fazia com que o doente se sentisse "desligado da sociedade" (idem), além da concepção de que o tratamento médico era insuficiente para assegurar a eficácia do retorno à vida no meio social. Esse serviço, assim como os demais em meio aberto, contribuiria para a realização do controle periódico do egresso, pois ele próprio procuraria esses serviços caso sentisse a manifestação de algum sintoma. Esse processo também geraria benefícios econômicos já que seriam evitadas as reinternações (idem).

\section{O cuidado em liberdade: uma defesa de ontem e de hoje}

Neste estudo, procuramos resgatar algumas considerações propostas pelos higienistas relativas às modalidades de tratamento em meio aberto no Brasil, no início do século XX. Embora as iniciativas debatidas nesse período não visassem à substituição do tratamento em regime fechado nem tivessem como escopo fundamental a preocupação com a autonomia e o resgate da cidadania daqueles que padeciam psiquicamente, a comparação entre as concepções dos higienistas e as conquistas atuais da reforma psiquiátrica fomentam reflexões.

Aliás, a leitura das propostas higienistas remete-nos, quase inevitavelmente, aos programas e serviços da atualidade, por exemplo, a oferta de auxílio material aos egressos proposta pelos higienistas pode ser associada ao Programa de Volta para Casa (PVC) ${ }^{14}$ e a modalidade de assistência familiar aos Serviços Residenciais Terapêuticos (SRTs). ${ }^{15}$ Salvaguardadas as devidas diferenças históricas e seus objetivos, o que esta pesquisa mostra é a existência de um ponto em comum entre essas modalidades de atendimento ao longo do tempo, isto é, a construção de meios e alternativas, com a finalidade de efetivar o cuidado em liberdade. Conquanto não utilizassem a nomenclatura "rede", algumas propostas higienistas continham a preocupação em ampliar as intervenções para além dos cuidados médicos. Destacamos,

${ }^{14}$ O PVC, instituído pela da Lei n. 10.708 de 31 de julho (Brasil, 2003), prevê o pagamento de um auxílio reabilitação psicossocial ao portador de transtorno mental egresso de internações com tempo superior a dois anos.

${ }^{15}$ Os SRTs são serviços criados para acolher pessoas egressas de longa internação psiquiátrica e hospitais de custódia e devem se constituir em casas inseridas na comunidade visando a inserção social de seus moradores, segundo a Portaria n. 106 de 11 de fevereiro (Brasil, 2000). 
assim, que algumas décadas antes da consolidação do movimento da reforma psiquiátrica, alguns higienistas já apregoavam que a situação das pessoas consideradas doentes mentais não seria resolvida por meio de intervenções isoladas e independentes.

$\mathrm{O}$ resgate histórico empreendido nesta pesquisa leva-nos a concluir que os desafios e as tentativas de solução no campo da saúde mental não são novos. Observamos que o movimento higienista e sua vertente voltada à higiene mental, em estudos sobre a história da saúde mental no país são, muitas vezes, abordados como um fator reforçador do modelo asilar de atendimento, sobretudo devido às suas concepções de cunho organicista, dentre elas a defesa da teoria da degenerescência hereditária. É fato que este viés do movimento não pode ser negado, pois alguns higienistas, ao mesmo tempo que defendiam os serviços abertos, apregoavam a necessidade de internação em manicômios ou de esterilização a fim de evitar procriação, em alguns casos, posicionamento observado, por exemplo, nas produções de Lopes (1930, 1933).

Entretanto, a contribuição deste estudo consiste em apresentar um lado pouco conhecido ou pouco divulgado desse movimento que, apesar de não questionar a existência dos manicômios, vislumbrou, ainda nas primeiras décadas do século XX, a possibilidade do tratamento em liberdade.

\section{Referências}

Amarante, P. D. C. (1995). Novos sujeitos, novos direitos: o debate em torno da reforma psiquiátrica. Cadernos de Saúde Pública, 11(3), 491-494.

Amarante, P. D. C. (1996). O homem e a serpente: outras histórias para a loucura e a psiquiatria. Rio de Janeiro: Editora Fiocruz.

Anônimo (1949). Assistência aos egressos de hospital psiquiátrico. Boletim de Higiene Mental, 5(58), 2.

Boarini, M. L. (2003). Higiene e raça como projetos: higienismo e eugenismo no Brasil. Maringá: Eduem.

Brasil. Decreto n. 1.132 (1903, 22 de dezembro). Organiza a Assistência a Alienados. Recuperado de <http://www2.camara.leg.br/legin/fed/decret/1900-1909/ decreto-1132-22-dezembro-1903-585004-publicacaooriginal-107902-pl.html>.

Brasil. Decreto n. 8.834 (1911, 11 de julho). Organiza a Assistência a Alienados. Recuperado de <http:/www2.camara.leg.br/legin/fed/decret/1910-1919/decreto-8834-11-julho-1911-507693-publicacaooriginal-1-pe.html $>$. 


\section{HISTÓRIA DA PSIQUIATRIA}

Brasil. Portaria n. 106. (2000, 11 de fevereiro). Institui os Serviços Residenciais Terapêuticos. Brasília, DF: Ministério da Saúde. Recuperado de <http://portalsaude. saude.gov.br/images/pdf/2015/marco/10/PORTARIA-106-11-FEVEREIRO-2000. pdf>.

Brasil. Lei n. 10.708 (2003, 31 de julho). Institui o auxílio-reabilitação psicossocial para pacientes acometidos de transtornos mentais egressos de internações. Brasília, DF: Presidência da República: Casa Civil. Subchefia para Assuntos Jurídicos. Recuperado de <http://planalto.gov.br/ccivil_03/Leis/2003/L10.708.htm>.

Costa, J. F. (2007). História da psiquiatria no Brasil: um corte ideológico (5 $5^{\mathrm{a}} \mathrm{ed}$. rev.). Rio de Janeiro: Garamond.

Cunha, M. C. P. (1986). O espelho do mundo: Juquery, a história de um asilo. Rio de Janeiro: Paz e Terra.

Godoy, J. (1955). Tese apresentada ao Congresso Médico realizado na Cidade de Rio Grande, no ano de 1929, e a criação do Pôsto de Psicopatas nesta capital. In J. Godoy (Org.), Psiquiatria no Rio Grande do Sul (pp. 101-111). [S. 1.: s. n.].

Jorge, M. A. S. (1997). Engenho dentro de casa: sobre a construção de um serviço de atenção diária em saúde mental. Dissertação de Mestrado, Fundação Oswaldo Cruz, Escola Nacional de Saúde Pública, Rio de Janeiro.

Liga Brasileira de Hygiene Mental. (1925). Archivos Brasileiros de Hygiene Mental, 1(1), 223-234.

Lopes, E. (1930). Assistencia Familiar de alienados (Resenha). Archivos Brasileiros de Hygiene Mental, 3(4), 255-257. Recuperado de $<$ http://old.ppi.uem.br/gephe/ ABHM/ABHMAno3N4Abr1930.pdf>.

Lopes, E. (1933). A alta tardia dos heredo-psychopathas por motivo de ordem eugênica: Subsidio para a nossa lei de assistencia a psychpathas. Archivos Brasileiros de Hygiene Mental, 6(4), 277-289. Recuperado de $<$ http://old.ppi.uem.br/gephe/ ABHM/ABHMAno6N4OutDez1933.pdf>.

Moreira, J. (2016). In Dicionário Histórico-Biográfico das Ciências da Saúde no Brasil (1832-1930). Recuperado de <http://www.dichistoriasaude.coc.fiocruz.br/ $\mathrm{iah} / \mathrm{pt} / \mathrm{verbetes} /$ morjul.htm>.

Pacheco e Silva, A. C. (1928). Necessidade da criação de serviços abertos para a internação voluntária de psicopatas, de ambulatórios, dispensários e de uma clínica psiquiátrica em São Paulo. Arquivos Paulistas de Higiene Mental 1(1), 1-6. Recuperado de $<$ http://old.ppi.uem.br/gephe/APHM/APHMAno1N1Jun1928parte1. pdf $>$.

Pacheco e Silva, A. C. (1944). O serviço social na assistência aos doentes da mente. Boletim de Higiene Mental, 1(4), 1-3.

Peixoto, J. A. (2016). In Dicionário Histórico-Biográfico das Ciências da Saúde no Rev. Latinoam. Psicopat. Fund., São Paulo, 19(4), 737-750, dez. 2016 
Brasil (1832-1930). Recuperado de < http://www.dichistoriasaude.coc.fiocruz.br/ iah/pt/verbetes/julafpeix.htm>.

Rezende, G. (1929). Patronato dos egressos dos manicômios. Archivos Brasileiros de Hygiene Mental, 2(2), 64-67.

Rezende, G. (1932). Assistencia hetero-familiar na Colonia de Psicopatas (mulheres) no Engenho de Dentro. In W. Pires (Org.), Anais da Assistencia a Psicopatas (pp. 133-138). Rio de Janeiro: Imprensa Nacional. Recuperado de <http://bvsms.saude. gov.br/bvs/publicacoes/cd05_03.pdf $>$.

Riedel, G. K. In Base Arch.: Base de dados do acervo arquivístico da Casa de Oswaldo

Cruz. Recuperado de $<\mathrm{http}$ ://arch.coc.fiocruz.br/index.php/gustavo-kohler-riedel $>$.

Santos, A. C. S. (1931). Da clínica psychiatrica e do ambulatório de hygiene mental. Tese de Doutorado, Faculdade de Medicina de São Paulo, São Paulo.

Silva, R. P. (2014). As ciências de Afrânio Peixoto: higiene, psiquiatria e medicina legal (1892-1935). Tese de Doutorado, Programa de Pós-Graduação em História das Ciências e da Saúde, Casa Oswaldo Cruz/Fiocruz, Rio de Janeiro.

Wadi, Y. M. (2002). Palácio para guardar doidos: uma história das lutas pela construção do hospital de alienados e da psiquiatria no Rio Grande do Sul. Porto Alegre: Editora da UFRGS.

\section{Resumos}

(The open mental health services in Brazil: The care in freedom from the perspective of hygienists)

This study aims to present considerations from hygienists about the open services for mental health developed in Brazil at the beginning of the twentieth century. We analyze publications from the Archivos Brasileiros de Hygiene Mental and other materials (1925-1955), and find that the possibility of treating people in psychic suffering in freedom (defended by the current psychiatric reform) was already glimpsed by hygienists, however, in a period during which mental health assistance was hegemonically based on isolation within psychiatric institutions.

Key words: Mental hygiene, history of psychiatry, psychiatric reform, mental health services

(Les Services Ouverts de Santé Mentale au Brésil: les soins en liberté du point de vue des hygiénistes)

Le but de cette étude est de présenter les réflexions de quelques médecins hygiénistes sur les services ouverts de santé mentale développés au Brésil au début du 


\section{HISTÓRIA DA PSIQUIATRIA}

XXème siècle. On a analysé des publications des Archives Brésiliennes d'Hygiène Mentale ainsi que d'autres documents (1925-1955) et on a vérifié que la possibilité d'un traitement en liberté (soutenu par l'actuelle réforme psychiatrique) était déjà envisagée par les hygiénistes lors d'une période pendant laquelle les soins de santé mentale était basés, de façon hégémonique, sur l'isolement des patients dans les établissements psychiatriques.

Mots clés: Hygiène mentale, histoire de la psychiatrie, reforme psychiatrique, Services de Santé Mentale

(Los servicios abiertos de salud mental en Brasil: el cuidado en libertad desde la perspectiva de los higienistas)

El objetivo de esta investigación es presentar las consideraciones de algunos médicos higienistas sobre los servicios abiertos de salud mental desarrollados en Brasil a comienzos del siglo XX. Analizamos publicaciones de los Archivos Brasileños de Higiene Mental y otros materiales (1925-1955), y verificamos que la posibilidad del cuidado en libertad (principio defendido por la actual Reforma Psiquiátrica( ya había sido vislumbrado por los higienistas, en un periodo en el que la asistencia estaba, en su gran mayoría, fundamentada en el aislamiento en instituciones psiquiátricas.

Palabras clave: Higiene mental, historia de la psiquiatría, reforma psiquiátrica,

Servicios de salud mental

(Ambulante psychiatrische Pflege in Brasilien: Die ambulante Betreuung aus der Perspektive der Hygienisten)

Ziel der vorliegenden Studie ist es, die Ansichten einiger ,Hygienebeauftragter Ärzte" zur ambulanten psychiatrischen Pflege zu Beginn des zwanzigsten Jahrhunderts in Brasilien zu erläutern. Wir analysierten Publikationen der 'Archivos Brasileiros de Hygiene Mental' und andere Quellen aus jener Zeit (1925-1955) und stellten fest, dass einige „Hygienebeauftragte Ärzte“ die Möglichkeit der ambulante Pflege (welche heute von der psychiatrischen Reform vertreten wird) bereits in Erwägung zogen, zu einer Zeit in der die psychiatrische Pflege ausschliesslich auf die Absonderung der Patienten in psychiatrischen Anstalten basierte.

Schlüsselwörter: Mentale Hygiene, Geschichte der Psychiatrie, Psychiatrische Reform, Psychiatrische Pflege

（在巴西开放的精神健康服务：由卫生专家的角度观看自由看护）

这项研究的目的是为了发表几位卫生专家对于巴西二十世纪初发展的开 放精神健康服务的审议。经由验证「巴西人精神健康档案」与其它发布资料

（1925-1955），我们证实了当时的卫生专家，已经微见了对于饱受精神病痛 
的患者，能够使用自由护理的可能性（由目前精神料改革所主张），在那个时 期，基于精神保健的唯一准则，精神病院都需要被完全隔离。

关键词：精神健康；精神料历史；精神料改革；精神健康服务。

Citação/Citation: Frazatto, C. F., Beltrame. M. M. (2016, dezembro). Os Serviços Abertos de Saúde Mental no Brasil: o cuidado em libverdade na perspectiva dos higienistas. Revista Latinoamericana de Psicopatologia Fundamental, 19(4), 737-750.

Editores do artigo/Editors: Profa. Dra. Ana Maria G. Raimundo Oda e Prof. Dr. Paulo Dalgalarrondo.

Recebido/Received: 07.08.2016/ 08.07.2016 Aceito/Accepted: 18.12.2016/ 12.18.2016

Copyright: (C) 2009 Associação Universitária de Pesquisa em Psicopatologia Fundamental/ University Association for Research in Fundamental Psychopathology. Este é um artigo de livre acesso, que permite uso irrestrito, distribuição e reprodução em qualquer meio, desde que o autor e a fonte sejam citados / This is an open-access article, which permits unrestricted use, distribution, and reproduction in any medium, provided the original authors and sources are credited.

Financiamento/Funding: Pesquisa financiada pelo Conselho Nacional de Desenvolvimento Científico e Tecnológico - CNPq / Research funded by Conselho Nacional de Desenvolvimento Científico e Tecnológico - CNPq

Conflito de interesses/Conflict of interest: As autoras declaram que não há conflito de interesses / The authors have no conflict of interest to declare.

\section{Carina Furlaneto Frazatto}

Doutoranda em Psicologia Social pela Pontifícia Universidade Católica de São Paulo PUC-SP (São Paulo, SP, Br); Bolsista CNPq. (Brasília, DF, Br)

Rua Santa Efigênia, 1333 - Centro

87080-250 Marialva, PR, Br

caryfrazatto@hotmail.com.

\section{Marina Maria Beltrame}

Doutoranda em Psicologia, na Universidade Estadual de Maringá - UEM (Maringá, PR, $\mathrm{Br})$.

Rua Nassib Haddad, 850 - Zona 05

87015-270 Maringá, PR, Br.

marimaria11@yahoo.com.br.

This is an open-access article, which permits unrestricted use, distribution, 